\title{
ON SOME CLASSES OF INTEGRO-DIFFERENTIAL EQUATIONS ON THE HALF-LINE AND RELATED OPERATOR FUNCTIONS
}

\author{
V. V. VLASOV
}

\begin{abstract}
We study integro-differential equations with unbounded operator coefficients on the half-line. The symbols of these equations are polynomial operator pencils perturbed by operator functions holomorphic (regular) in the left half-plane.

Results concerning the Fredholm property of initial-boundary value problems for these equations on the half-line and the properties of their exponential (elementary) solutions are established.

The properties of the derived chains constructed from the root vectors of the operator functions that are the symbols of these equations are considered.
\end{abstract}

\section{INTRODUCTION}

The main subject of the present paper is initial-boundary value problems for functional-differential equations whose principal symbols are polynomial operator pencils perturbed by operator functions holomorphic in the left half-plane.

We establish the Fredholm property of these problems in the Sobolev spaces on the half-line. The proof of the main result is based on the reduction of these problems to integral equations of Wiener-Hopf type on the half-line with the subsequent proof of the Fredholm solvability of the latter. The results can be applied when studying the completeness and minimality of the system of exponential solutions of these equations as well as the multiple minimality of the root vectors of the operator functions that are the symbols of these equations.

It is worth noting that operator functions that are polynomial operator pencils perturbed by operator functions holomorphic outside a disk (or in a half-plane) were studied for a number of years by Radzievskii. For example, the papers [18, [20, [21], and [24] deal with the multiple completeness of the root vectors and associated derived chains, and the papers [19], 22], and 23] study the minimality and the basis property of the derived chains associated with the root vectors of operator functions. Of the papers concerned with the completeness of derived chains, we especially mention the comprehensive survey 24 .

Note also that Radzievskii's results on the estimates for the above-mentioned operator functions prove useful when studying the asymptotic behavior of strong solutions of integro-differential equations naturally associated with these operator functions.

The peculiarity of the problems considered in the present paper is that for $n \geq 2$ we study not only the initial value problem (the Cauchy problem) but also a boundary value problem on the half-line. Thus, the number of conditions posed at $t=0$ can be less than the order $n$ of the equation. However, in this case we consider the solutions bounded (in the integral sense) as $t \rightarrow+\infty$.

2010 Mathematics Subject Classification. Primary 47G20; Secondary 34K30, 47A56, 34K12.

Key words and phrases. Integro-differential equation, operator function, exponential solution.

Supported by RFBR grants nos. 11-01-00790 and 11-01-12115-ofi-m-2011 and by State Support of Leading Scientific Schools grant no. NSh-7322.2010.1. 
We also state results on the nonexistence of nontrivial (nonzero) solutions of homogeneous functional-differential equations decaying more rapidly than any exponential (the Phragmén-Lindelöf principle), on the dichotomy of solutions, on the minimality of the system of exponential solutions, and on the multiple minimality of the root vectors of the operator functions that are the symbols of the equations studied here.

The paper consists of an introduction, a section containing definitions, notation, and statements of the solvability results, a section containing the proofs of the main results on the Fredholm solvability, and a section in which the spectral results are stated. A scheme of proof of these results is given with some explanations.

In the closing section, we give some remarks and a brief comment.

\section{Definition, notation, And Statements of the main Results}

Let $\mathfrak{H}$ be a separable Hilbert space, and let $A$ be a self-adjoint positive operator with bounded inverse on $\mathfrak{H}$. We make the domain $D\left(A^{\beta}\right)(\beta>0)$ a Hilbert space $\mathfrak{H}_{\beta}$ by equipping $D\left(A^{\beta}\right)$ with the norm $\|\cdot\|_{\beta}=\left\|A^{\beta} \cdot\right\|$, which is equivalent to the graph norm.

By $W_{2}^{n}\left(\mathbb{R}_{+}, A^{n}\right)$ we denote the Sobolev space of vector functions ranging in $\mathfrak{H}$ such that $A^{n-j} v^{(j)} \in L_{2}\left(\mathbb{R}_{+}, \mathfrak{H}\right), j=0,1, \ldots, n$, equipped with the norm

$$
\|\varphi\|_{W_{2}^{n}} \equiv\left(\int_{0}^{\infty}\left(\left\|\varphi^{(n)}(t)\right\|_{\mathfrak{H}}^{2}+\left\|A^{n} \varphi(t)\right\|_{\mathfrak{H}}^{2}\right) d t\right)^{1 / 2} .
$$

For more detail about this space, see the monograph [14, Ch. I].

On the half-line $\mathbb{R}_{+}=(0,+\infty)$, consider the equation

$$
\mathfrak{U} u \equiv G\left(\frac{d}{d t}\right) u(t)+(H u)(t)+(K u)(t)=f(t),
$$

where $G(\lambda)$ is an operator pencil of the form

$$
G(\lambda)=\lambda^{n} G_{0}+\lambda^{n-1} G_{1} A+\cdots+G_{n} A^{n}
$$

with bounded operators $G_{j}$ on $\mathfrak{H}$ and the operators $H$ and $K$ are defined on the space $W_{2}^{n}\left(\mathbb{R}_{+}, A^{n}\right)$, and map it into $L_{2}\left(\mathbb{R}_{+}, \mathfrak{H}\right)$ according to the rule

$$
\begin{aligned}
& (H \varphi)(t)=Q_{+}\left(F^{-1}\left(S(i \mu) F\left(R\left(A^{n} \varphi\right)\right)(\mu)\right)\right)(t), \\
& (K \varphi)(t)=\sum_{j=1}^{n} \int_{0}^{+\infty} K_{j}(t-s) A^{j} \varphi^{(n-j)}(s) d s
\end{aligned}
$$

here the $K_{j}(t)$ are operator functions ranging in the set of compact operators on $\mathfrak{H}$ and Bochner integrable on the line $\mathbb{R}=(-\infty,+\infty) ; F$ and $F^{-1}$ are the direct and inverse Fourier transform, respectively; $R$ is the operator of extension of functions by zero from $\mathbb{R}_{+}$to $\mathbb{R}$ (i.e., $(R \varphi)(t)=\varphi(t), t \geq 0$, and $\left.(R \varphi)(t)=0, t<0\right) ; Q_{+}$is the operator of restriction to the positive half-line (i.e., $\left.\left(Q_{+} \varphi\right)(t)=\varphi(t), t>0\right)$; and $f(t) \in L_{2}\left(\mathbb{R}_{+}, \mathfrak{H}\right)$ is a vector function.

We assume that the operator function $S(i \mu)$ satisfies one of the following conditions.

(A1) The operator function $S(i \mu)$ is continuous and bounded on $\mathbb{R}$ in the operator norm, and

$$
\lim _{m \rightarrow \infty} \sup _{\mu \in \mathbb{R}}\left\|S(i \mu)-Q_{m} S(i \mu) Q_{m}\right\|=0 ;
$$

here $Q_{m}$ is the orthogonal projection onto the subspace spanned by the first $m$ eigenvectors $\left\{e_{j}\right\}_{j=1}^{m}$ of $A$. The eigenvalues $a_{j}\left(A e_{j}=a_{j} e_{j}\right)$ of $A$ are numbered in ascending order with regard to multiplicities $\left(0<a_{1} \leq a_{2} \leq \cdots\right)$. 
(A2) The operator function $S(i \mu)$ is continuous on $\mathbb{R}$ in the operator norm, ranges in the set of compact operators on $\mathfrak{H}$, and satisfies the relation

$$
\|S(i \mu)\| \rightarrow 0 \text { as }|\mu| \rightarrow+\infty .
$$

The variable $\mu$ in (2) and in what follows is the variable of the Fourier transform.

Let us present a result on the solvability of some initial-boundary value problems on the half-line $\mathbb{R}_{+}$for Eq. (1) for the case in which the pencil $G(\lambda)$ has the form

$$
\begin{gathered}
G(\lambda)=P_{0}(\lambda)+P_{1}(\lambda) \\
P_{0}(\lambda)=\prod_{j=1}^{m}\left(\lambda I-\omega_{j} A\right)^{s_{j}}, \quad P_{1}(\lambda)=\sum_{j=1}^{n} \lambda^{n-j} B_{j} A^{j} .
\end{gathered}
$$

Here the $B_{j}$ are arbitrary compact operators on $\mathfrak{H}$; the $\omega_{j}$ are complex numbers such that $\arg \omega_{p} \neq \arg \omega_{q}$ for $p \neq q, \operatorname{Re} \omega_{j}<0$ for $j=1,2, \ldots, h, \operatorname{Re} \omega_{j}>0$ for $j=h+1, \ldots, m$, and $n=s_{1}+s_{2}+\cdots+s_{m}$.

Let $r_{j}$ be integers satisfying the inequalities

$$
0 \leq r_{1}<r_{2}<\cdots<r_{k}<n, \quad k=s_{1}+s_{2}+\cdots+s_{h} .
$$

Using the numbers $r_{l}, l=1,2, \ldots, k$, and $\omega_{p}, p=1,2, \ldots, h$, we construct a matrix $\left\|\nu_{l, j}\right\|_{l, j=1}^{k}$ as follows: for an index $j$ satisfying the inequality $s_{0}+s_{1}+\cdots+s_{p-1}<j \leq$ $s_{1}+s_{2}+\cdots+s_{p}\left(p=1,2, \ldots, h ; s_{0}=0\right)$, set $\pi_{j}=j-s_{0}-s_{1}-\cdots-s_{p-1}-1$ and define

$$
\nu_{l, j}=\left.\frac{d^{\pi_{j}}}{d \lambda^{\pi_{j}}}\left(\lambda^{r_{l}}\right)\right|_{\lambda=\omega_{p}}, \quad l, j=1,2, \ldots, k .
$$

Let $B: W_{2}^{n}\left(\mathbb{R}_{+}, A^{n}\right) \rightarrow L_{2}\left(\mathbb{R}_{+}, \mathfrak{H}\right) \oplus\left(\bigoplus_{m=1}^{k} \mathfrak{H}_{n-r_{m}-1 / 2}\right)$ be the operator defined by

$$
B \varphi \equiv\left(\mathfrak{U} \varphi, \varphi^{\left(r_{1}\right)}(+0), \varphi^{\left(r_{2}\right)}(+0), \ldots, \varphi^{\left(r_{k}\right)}(+0)\right) .
$$

We introduce the operator functions

$$
\begin{gathered}
L(\lambda)=G(\lambda)+S(\lambda) A^{n}+T(\lambda) \\
T(\lambda)=\sum_{j=1}^{n} \lambda^{n-j} T_{j}(\lambda) A^{j}, \quad T_{j}(\lambda)=\int_{-\infty}^{+\infty} e^{-\lambda t} K_{j}(t) d t .
\end{gathered}
$$

Theorem 1. Assume that the pencil $G(\lambda)$ can be represented in the form (4); the operator function $\left(L(\lambda) P_{0}^{-1}(\lambda)\right)^{-1}$ is bounded on the imaginary axis; the operator function $S(i \mu)$ satisfies condition (A1); and $\operatorname{det}\left\|\nu_{l, j}\right\| \neq 0$. Then the operator $B$ is Fredholm.

Definition. A strong solution of (11) is a vector function $u \in W_{2}^{n}\left(\mathbb{R}_{+}, A^{n}\right)$ satisfying Eq. (1) almost everywhere on $\mathbb{R}_{+}$.

Theorems 24 deal with the invertibility of the operator $B$ in the special cases of $n=1$ and $n=2$.

Theorem 2. Suppose that the assumptions of Theorem 1 hold with $n=1, r_{1}=0$, $\operatorname{Re} \omega_{1}<0$, the operator function $S(\lambda)$ is regular in the half-plane $\Pi(\varkappa)=\{\lambda$ : $\operatorname{Re} \lambda>$ $-\varkappa\}, \varkappa>0,\|S(\lambda)\| \rightarrow 0$ as $|\lambda| \rightarrow+\infty, \lambda \in \Pi(\varkappa), K_{1}(t)=0$ for $t<0$, and $e^{\delta t}\left\|K_{1}(t)\right\| \in$ $L_{2}\left(\mathbb{R}_{+}\right), \delta>0$. Then $\operatorname{Ker} B=\{0\}$, the operator $B$ has a bounded inverse acting from $\operatorname{Im} B \subset L_{2}\left(\mathbb{R}_{+}, \mathfrak{H}\right) \oplus \mathfrak{H}_{1 / 2}$ to $W_{2}^{1}\left(\mathbb{R}_{+}, A\right)$, and the inequality

$$
\|u\|_{W_{2}^{1}} \leq d\left(\|\mathfrak{U} u\|_{L_{2}}^{2}+\|u(+0)\|_{1 / 2}^{2}\right)^{1 / 2}
$$

holds with a constant $d$ independent of $(\mathfrak{U} u, u(+0)) \in \operatorname{Im} B$. 
Note that, under our assumptions, $G(\lambda)$ is a Keldysh pencil in unbounded notation. (The polynomial $P_{0}(\lambda)$ is allowed to have multiple roots.)

Remark 1. For $\omega_{1}=-1$, the assumptions of Theorem 2 can be weakened. Namely, the operator $A$ in Eq. (11) can be replaced by a normal operator $C$ with compact inverse whose spectrum lies in the sector $\{\lambda:|\arg \lambda|<\theta\}, 0 \leq \theta<\pi / 2$. In this case, the space $W_{2}^{1}\left(\mathbb{R}_{+}, A\right)$ is defined with the use of the operator $A=\left(C^{*} C\right)^{1 / 2}$.

Theorem 3. Suppose that the assumptions of Theorem 1 are satisfied with $n=1, \omega_{1}=$ $-1, r_{1}=0, \operatorname{Re} B_{1} \geq 0$, the operator function $S(i \mu)$ satisfies the condition $\operatorname{Re}(S(i \mu) x, x) \geq$ 0 for any $x \in \mathfrak{H}$ and $\mu \in \mathbb{R}$, and $K_{1}(t) \equiv 0$. Then the assertion of Theorem 2 holds.

Theorem 4. Suppose that the assumptions of Theorem 1 are satisfied with $n=2$, $\omega_{1}=-1, \omega_{2}=1, r_{1}=0, k=1, B_{1}=0, \operatorname{Re} B_{2} \leq 0, K_{j}(t)=0, j=1,2$, and the operator function $S(i \mu)$ satisfies the condition $\operatorname{Re}(S(i \mu) x, x) \leq 0$ for any $x \in \mathfrak{H}$ and $\mu \in \mathbb{R}$. Then $\operatorname{Ker} B=0$ and the operator $B$ has a bounded inverse acting from $\operatorname{Im} B \subseteq L_{2}\left(\mathbb{R}_{+}, \mathfrak{H}\right) \oplus \mathfrak{H}_{3 / 2}$ to $W_{2}^{2}\left(\mathbb{R}_{+}, A^{2}\right)$.

Let us present an assertion describing the behavior of the operator function $L^{-1}(\lambda)$ in the special case of $n=1$.

Lemma 1. Suppose that the assumptions of Theorem 2 are satisfied. Then the spectrum of the operator function $L(\lambda)$ in the right half-plane consists of finitely many characteristic numbers of finite algebraic multiplicity, which are finite rank poles of the resolvent $L^{-1}(\lambda)$, and the estimate

$$
|\lambda|\left\|L^{-1}(\lambda)\right\|+\left\|A L^{-1}(\lambda)\right\| \leq c
$$

with constant $c$ independent of $\lambda$ holds in the domain $\Omega \equiv\{\lambda: \operatorname{Re} \lambda>0\} \cup\{\lambda:|\lambda|>R\}$, where $R$ is a sufficiently large positive number.

Remark 2. In particular, the equation

(5) $\frac{d u}{d t}(t)+\left(I+B_{1}\right) A u(t)+\int_{0}^{t} K_{1}(t-s) A u(s) d s+\sum_{j=1}^{r} \theta\left(t-h_{j}\right) C_{j} A u\left(t-h_{j}\right)=f(t)$

can be reduced to an equation of the form (11). Here $B_{1}$ and $C_{j}$ are compact operators on $\mathfrak{H}, \theta(\cdot)$ is the Heaviside step function, and the $h_{j}$ are real numbers such that $0 \leq h_{1}<$ $h_{2}<\cdots<h_{r}$; moreover, the operator function $S(i \mu)$ is given by

$$
S(i \mu)=\sum_{j=1}^{r} e^{i \mu h_{j}} C_{j}
$$

Note that Eq. (5) is a version of the heat equation in media with memory. Such media are studied in a number of papers (see [5]-7] and the references therein). We also point out that an equation of the form (5), as well as a more general equation with variable operator coefficients in weighted Sobolev spaces on the half-line, was studied in [5], 6], and [7.

The norms of operators on the spaces $L_{2}(\mathbb{R}, \mathfrak{H})$ and $L_{2}\left(\mathbb{R}_{+}, \mathfrak{H}\right)$ will be denoted by $\|\cdot \mid\|$ and $\||\cdot|\|_{+}$, respectively, in what follows.

\section{Proof of the MAin ASSERTions}

Let us present the proof of Theorem 1 for the case in which $s_{j}=1, j=1,2, \ldots, n$.

The proof of the Fredholm property of $B$ coincides with that of the Fredholm property of Eq. (1) with the conditions

$$
u^{\left(r_{j}\right)}(+0)=\varphi_{j}, \quad j=1,2, \ldots, k .
$$


One can verify that the equation

$$
P_{0}\left(\frac{d}{d t}\right) u(t)=v(t), \quad v \in L_{2}\left(\mathbb{R}_{+}, \mathfrak{H}\right),
$$

is satisfied by the vector function

$$
u_{0}(t)=\int_{0}^{+\infty} G_{0}(t-s) v(s) d s, \quad G_{0}(t-s)=\frac{1}{2 \pi i} \int_{-i \infty}^{+i \infty} P_{0}^{-1}(\lambda) e^{\lambda(t-s)} d \lambda .
$$

Then the general solution of problems (7), (6) can be represented in the form

$$
u(t)=u_{0}(t)+\sum_{m=1}^{k} \sum_{j=1}^{k} \alpha_{m j} A^{-r_{j}} e^{\omega_{m} t A}\left(\varphi_{j}-u^{\left(r_{j}\right)}(+0)\right),
$$

where $\alpha_{m j}=b_{m j} / \Delta, b_{m j}$ is the cofactor of the $(m, j)$ th entry of the matrix $\left\|\omega_{i}^{r_{j}}\right\|_{i, j=1}^{k}$, and $\Delta=\operatorname{det}\left\|\omega_{i}^{r_{j}}\right\|_{i, j=1}^{k}$.

On the other hand, according to (1), one has

$$
\begin{aligned}
P_{0}\left(\frac{d}{d t}\right) u(t)=v(t)=-P_{1}\left(\frac{d}{d t}\right) u(t)- & \sum_{j=1}^{n} \int_{0}^{+\infty} K_{j}(t-s) A^{j} u^{(n-j)}(s) d s \\
& -Q_{+}\left(F^{-1}\left(S(i \mu) F\left(R\left(A^{n} u\right)\right)\right)\right)(t)+f(t) .
\end{aligned}
$$

This, together with relations (8) $-(10)$, gives the equation

$$
v+\sum_{j=1}^{7} D_{j} v=F
$$

for the function $v$, in which the operators $D_{j}$ are defined as follows:

$$
\begin{aligned}
& D_{1} v=\int_{0}^{+\infty} V_{1}(t-s) v(s) d s, \quad V_{1}(t-s)=P_{1}\left(\frac{d}{d t}\right) G_{0}(t-s), \\
& D_{2} v=\int_{-\infty}^{+\infty} \sum_{j=1}^{n} K_{j}(t-s)\left(\int_{0}^{+\infty} A^{j} G_{0}^{(n-j)}(s-\zeta) v(\zeta) d \zeta\right) d s \\
& D_{3} v=-\int_{0}^{+\infty} \sum_{j=1}^{n} K_{j}(t+s)\left(\int_{0}^{+\infty} A^{j} G_{0}^{(n-j)}(-s-\zeta) v(\zeta) d \zeta\right) d s \\
& D_{4} v=Q_{+}\left(F^{-1}\left(\left(S(i \mu) A^{n} P_{0}^{-1}(i \mu)\right) F(R v)(\mu)\right)\right)(t) \\
& D_{5} v=-Q_{+}\left(F^{-1}\left(S(i \mu) F\left(Q_{-}\left(\int_{0}^{+\infty} A^{n} G_{0}(s-\zeta) v(\zeta) d \zeta\right)\right)\right)\right)(t),
\end{aligned}
$$

where $Q_{-}$is the operator of restriction to the negative half-line (i.e., $\left(Q_{-} \varphi\right)(t)=0$ for $t>0$ and $\left(Q_{-} \varphi\right)(t)=\varphi(t)$ for $\left.t \leq 0\right)$,

$$
D_{6} v=\int_{0}^{+\infty} \sum_{p=1}^{n} K_{p}(t-s) A^{p} \frac{d^{n-p}}{d s^{n-p}} \widetilde{\Phi}(s) d s
$$

where $\widetilde{\Phi}(s)=\sum_{m=1}^{k} \sum_{j=1}^{k} \alpha_{m j} e^{\omega_{m} s A} A^{-r_{j}}\left(\int_{0}^{+\infty} G_{0}^{\left(r_{j}\right)}(-\zeta) v(\zeta) d \zeta\right)$,

$$
D_{7} v=Q_{+}\left(F^{-1}\left(S(i \mu) A^{n}\left(F\left(R Q_{+}(\widetilde{\Phi})\right)\right)\right)\right)(t)
$$


where

$$
\begin{gathered}
F(t)=f(t)-P_{1}\left(\frac{d}{d t}\right) \Psi(t)-\sum_{p=1}^{n} \int_{0}^{+\infty} K_{p}(t-s) A^{p}\left(\frac{d^{n-p}}{d s^{n-p}} \Psi(s)\right) d s \\
-Q_{+}\left(F^{-1}\left(S(i \mu) A^{n}(F(R \Psi))(\mu)\right)\right)(t), \\
\Psi(t)=\sum_{m=1}^{k} \sum_{j=1}^{k} \alpha_{m j} A^{-r_{j}} e^{\omega_{m} t A} \varphi_{j}, \quad t \in \mathbb{R}_{+} .
\end{gathered}
$$

We will need the following assertions.

Proposition 1. Let $K(t)$ be an operator function ranging in the set of bounded operators on $\mathfrak{H}$ and Bochner integrable on $\mathbb{R}\left(\right.$ i.e., $\left.K(t) \in L_{1}(\mathbb{R}, \mathfrak{H} \rightarrow \mathfrak{H})\right)$. Then the integral convolution operator

$$
\mathbb{K} \varphi=\int_{-\infty}^{+\infty} K(t-s) \varphi(s) d s
$$

is bounded on $L_{2}(\mathbb{R}, \mathfrak{H})$, and one has the inequality

$$
\|\mathbb{K}\| \leq \int_{-\infty}^{+\infty}\|K(t)\| d t .
$$

The proof of Proposition 1 can be found in the monograph [29, pp. 59-60].

Proposition 2. Under the above assumptions, the vector function $u_{0}(t)$ belongs to the space $W_{2}^{n}\left(\mathbb{R}_{+}, A^{n}\right)$.

The proof of the proposition follows from the estimate for $P_{0}^{-1}(\lambda)$ and from the fact that the Fourier transform is a unitary operator on $L_{2}(\mathbb{R}, \mathfrak{H})$.

By the trace theorem [14, p. 32], it follows from the inclusion $\varphi_{j} \in \mathfrak{H}_{n-r_{j}-1 / 2}, j=$ $1,2, \ldots, k$, that the second term on the right-hand side in (9) belongs to the space $W_{2}^{n}\left(\mathbb{R}_{+}, A^{n}\right)$ as well.

By using relations (7) and (10), we find that the Fredholm property of Eq. (11) implies that of the operator $B$.

Recall that an operator $B$ on a Banach space is said to be Fredholm if its range is closed and its null space and cokernel are finite-dimensional. In this case, we say that the equation $B x=y$ is $\Phi$-solvable.

First, let us give the scheme of proof of Theorem 1

Note that the leading part $\left(I+D_{1}+D_{2}+D_{4}\right) v=F$ of Eq. (11) is a Wiener-Hopf integral equation on the half-line $\mathbb{R}_{+}$. (The operators $D_{3}, D_{5}, D_{6}$, and $D_{7}$ are compact and do not affect the Fredholm property.) The main idea underlying the proof of the $\Phi$-solvability of Eq. (11) is as follows (just as in [10). First (Lemma 2), we establish the unique solvability of the equation

$$
\left(I+D_{1}+D_{2}+D_{4}\right) v=F
$$

on the entire line in the space $L_{2}(\mathbb{R}, \mathfrak{H})$. Then we rewrite this equation in the form of a system of equations in the orthogonal sum $L_{2}\left(\mathbb{R}_{+}, \mathfrak{H}\right) \oplus L_{2}\left(\mathbb{R}_{+}, \mathfrak{H}\right)$, where the operator to be studied is the upper entry on the main diagonal. Thus, to prove the Fredholm property (Lemma 3), it suffices to establish that the off-diagonal entries are compact operators on $L_{2}\left(\mathbb{R}_{+}, \mathfrak{H}\right)$. This is successively done in Proposition 4, Lemma 4, and Proposition 7 . Since the operators $D_{3}, D_{5}, D_{6}$, and $D_{7}$ are compact (see Propositions 8 and 9 and Lemma 5) on $L_{2}\left(\mathbb{R}_{+}, \mathfrak{H}\right)$, we see that the operator on the left-hand side in (11) is Fredholm; i.e., Eq. (11) is $\Phi$-solvable.

Let us proceed to the proof of the $\Phi$-solvability of Eq. (11). 
Lemma 2. Assume that the operator function $\left(L(\lambda) P_{0}^{-1}(\lambda)\right)^{-1}$ is bounded on the imaginary axis. Then the equation

$$
\begin{aligned}
& v(t)+\int_{-\infty}^{+\infty} V_{1}(t-s) v(s) d s \\
& \quad+\int_{-\infty}^{+\infty} \sum_{p=1}^{n} K_{p}(t-s)\left(\int_{-\infty}^{+\infty} A^{p} G_{0}^{(n-p)}(s-\zeta) v(\zeta) d \zeta\right) d s \\
& \quad+\left(F^{-1} S(i \mu) A^{n} P_{0}^{-1}(i \mu) F(v)\right)(t)=f(t)
\end{aligned}
$$

is uniquely solvable for an arbitrary function $f \in L_{2}(\mathbb{R}, \mathfrak{H})$.

To prove the lemma, it suffices to apply the Fourier transform to both parts of Eq. (12) and use the assumption of the lemma as well as the fact that the Fourier transform is a unitary operator on $L_{2}(\mathbb{R}, \mathfrak{H})$.

Lemma 3. Suppose that the assumptions of Lemma 2 are satisfied and the operator function $S(i \mu)$ satisfies condition (A1). Then the integral equation

$$
\mathfrak{N} v=v+D_{1} v+D_{2} v+D_{4} v=f
$$

is $\Phi$-solvable in $L_{2}\left(\mathbb{R}_{+}, \mathfrak{H}\right)$.

The proof of the lemma uses the following propositions.

Proposition 3. The integral operators

$$
D_{1}^{ \pm} v=\int_{0}^{+\infty} V_{1}( \pm t \pm s) v(s) d s, \quad t \in \mathbb{R}_{+},
$$

are compact in $L_{2}\left(\mathbb{R}_{+}, \mathfrak{H}\right)$.

The proof of this proposition for the operator $D_{1}^{+}$can be found in [9, Th. 1] and is based on the fact that, for each $m$, the operators

$$
D_{1, m}^{+} v=\int_{0}^{+\infty} V_{1}(t+s) Q_{m} v(s) d s
$$

are compact in $L_{2}\left(\mathbb{R}_{+}, \mathfrak{H}\right)$ and converge to $D_{1}^{+}$in the operator norm $\|\cdot\| \|_{+}$as $m \rightarrow \infty$ (see [9]).

The argument for the operator $D_{1}^{-}$is completely similar.

Proposition 4. The integral operators

$$
\begin{aligned}
& I_{p m}^{ \pm} v=\int_{0}^{+\infty} Q_{m} A^{p} G_{0}( \pm s \pm \zeta) Q_{m} v(\zeta) d \zeta, \quad s \in \mathbb{R}_{+}, \\
& J_{p m}^{ \pm} v=\int_{0}^{+\infty} Q_{m} K_{p}( \pm t \pm s) Q_{m} v(s) d s, \quad t \in \mathbb{R}_{+}, \quad p=1,2, \ldots, n,
\end{aligned}
$$

are compact in $L_{2}\left(\mathbb{R}_{+}, \mathfrak{H}\right)$.

The proof of Proposition 4 follows from [10, Th. 2.1], since the kernels $Q_{m} A^{p} G_{0}(t) Q_{m}$ and $Q_{m} K_{p}(t) Q_{m}$ of these integral operators lie in the space $L_{1}\left(\mathbb{R}, \mathbb{C}^{m} \times \mathbb{C}^{m}\right)$.

Proposition 5. Let $K_{p}(t)$ be an operator function ranging in the set of compact operators on $\mathfrak{H}$ and Bochner integrable on $\mathbb{R}$. Then

$$
\lim _{m \rightarrow \infty} \int_{-\infty}^{+\infty}\left\|K_{p}(t)-Q_{m} K_{p}(t) Q_{m}\right\| d t=0 .
$$


The proof of Proposition [5] is given in [7] and readily follows from Lebesgue's dominated convergence theorem and the fact that $\left\|Q_{m} K(t) Q_{m}-K(t)\right\| \rightarrow 0$ as $m \rightarrow \infty$ a.e. on $\mathbb{R}$.

Lemma 4. Let the assumptions of Proposition 5 be satisfied. Then the integral operators

$$
D_{2, p}^{ \pm} v=\int_{-\infty}^{+\infty} K_{p}( \pm t-\zeta)\left(\int_{0}^{+\infty} A^{p} G_{0}^{(n-p)}(\zeta \pm s) v(s) d s\right) d \zeta
$$

are compact in $L_{2}\left(\mathbb{R}_{+}, \mathfrak{H}\right)$.

The proof of the compactness of the operators $D_{2, p}^{ \pm}, p=1,2, \ldots, n$, follows a unified scheme. To be definite, let us establish that the operators $D_{2, p}^{-}$are compact. We represent $D_{2, p}^{-}$in the form $D_{2, p}^{-}=W_{p}+Y_{p}$, where

$$
\begin{aligned}
W_{p} v & =\int_{0}^{+\infty} K_{p}(-t-\zeta)\left(\int_{0}^{+\infty} A^{p} G_{0}^{(n-p)}(\zeta-s) v(s) d s\right) d \zeta \\
Y_{p} v & =\int_{0}^{+\infty} K_{p}(-t+\eta)\left(\int_{0}^{+\infty} A^{p} G_{0}^{(n-p)}(-\eta-s) v(s) d s\right) d \eta .
\end{aligned}
$$

Note that the operator

$$
W_{p m} v=J_{p m}^{-}\left(T_{p} v\right)=\int_{0}^{+\infty} Q_{m} K_{p}(-t-\zeta) Q_{m}\left(\int_{0}^{+\infty} A^{p} G_{0}^{(n-p)}(\zeta-s) v(s) d s\right) d \zeta
$$

is compact in $L_{2}\left(\mathbb{R}_{+}, \mathfrak{H}\right)$, because the operator $J_{p m}^{-}$is compact (see Proposition 4) and the operator

$$
T_{p} v=\int_{0}^{+\infty} A^{p} G_{0}^{(n-p)}(\zeta-s) v(s) d s
$$

is bounded in $L_{2}\left(\mathbb{R}_{+}, \mathfrak{H}\right)$ (see [30, Pt. 3]).

The sequence $W_{p m}$ converges to $W_{p}$ in the operator norm $\left\||\cdot \||_{+}\right.$as $m \rightarrow \infty$, which, according to Remark 1 and relation (13), is a consequence of the following chain of inequalities:

$$
\begin{aligned}
& \left\|\left(W_{p}-W_{p m}\right) v\right\|_{L_{2}} \leq \| \int_{0}^{+\infty}\left(K_{p}(-t-\zeta)-Q_{m} K_{p}(-t-\zeta) Q_{m}\right) \\
\times & \left(\int_{0}^{+\infty} A^{p} G_{0}^{(n-p)}(\zeta-s) v(s) d s\right) d \zeta\left\|_{L_{2}} \leq c\left(\int_{-\infty}^{+\infty}\left\|K_{p}(t)-Q_{m} K_{p}(t) Q_{m}\right\| d t\right)\right\| v \|_{L_{2}} .
\end{aligned}
$$

In turn, the compactness of the integral operator $Y_{p}$ follows from the fact that the operator

$$
Y_{p m} v=\int_{0}^{+\infty} Q_{m} K_{p}(-t+\eta) Q_{m}\left(\int_{0}^{+\infty} A^{p} G_{0}^{(n-p)}(-\eta-s) v(s) d s\right) d \eta
$$

is compact in $L_{2}\left(\mathbb{R}_{+}, \mathfrak{H}\right)$, being the composition of the bounded operator on $L_{2}\left(\mathbb{R}_{+}, \mathfrak{H}\right)$ with kernel $Q_{m} K(-t+\eta) Q_{m}$ and the compact operator

$$
I_{p m}^{-} v=\int_{0}^{+\infty} Q_{m} A^{p} G_{0}^{(n-p)}(-\eta-s) v(s) d s
$$

(see Proposition [5), and from the fact that the sequence $Y_{p m}$ converges to $Y_{p}$ in the operator norm $\left|\|\cdot \mid\|_{+}\right.$. 
This convergence, in accordance with Remark 1 and relation (13), follows from the inequalities

$$
\begin{aligned}
& \left\|\left(Y_{p}-Y_{p m}\right) v\right\|_{L_{2}} \leq \| \int_{0}^{+\infty}\left(K_{p}(-t+\eta)-Q_{m} K_{p}(-t+\eta) Q_{m}\right) \\
\times & \left(\int_{0}^{+\infty} A^{p} G_{0}^{(n-p)}(-\eta-s) v(s) d s\right) d \eta\left\|_{L_{2}} \leq c\left(\int_{-\infty}^{+\infty}\left\|K_{p}(t)-Q_{m} K_{p}(t) Q_{m}\right\| d t\right)\right\| v \|_{L_{2}} .
\end{aligned}
$$

Proposition 6. Let $Z(\mu)$ be a norm continuous $m \times m$ matrix function on $\mathbb{R}$ such that

$$
\|Z(\mu)\| \rightarrow 0 \quad \text { as }|\mu| \rightarrow+\infty .
$$

Then the integral operators

$$
Q_{ \pm} \int_{-\infty}^{+\infty} e^{i \mu t} Z(\mu)\left(\int_{0}^{+\infty} e^{ \pm i \mu s} v(s) d s\right) d \mu
$$

are compact in $L_{2}\left(\mathbb{R}_{+}, \mathbb{C}^{m}\right)$.

Note that the proof of this proposition uses some assertions in [10] (Theorem 2.1) as well as Theorem 2.2.1 in [16.

Indeed, by the assumption in the proposition and by [16, Th. 2.2.1], the matrix function $Z(\mu)$ can be approximated by an infinitely differentiable compactly supported matrix function $Z_{1}(\mu)$, so that

$$
\sup _{\mu \in \mathbb{R}}\left\|Z(\mu)-Z_{1}(\mu)\right\|_{\mathbb{C}^{m}}<\varepsilon .
$$

It readily follows from the last inequality and the fact that the Fourier transform is unitary in $L_{2}\left(\mathbb{R}, \mathbb{C}^{m}\right)$ that the integral operator to be studied can be approximated in the $L_{2}\left(\mathbb{R}, \mathbb{C}^{m}\right)$-operator norm by the integral operator

$$
Q_{ \pm} \int_{-\infty}^{+\infty} e^{i \mu t} Z_{1}(\mu)\left(\int_{0}^{+\infty} e^{ \pm i \mu s} v(s) d s\right) d \mu .
$$

In turn, the compactness of the latter integral operator follows from [11, Th. 2.1], because it can be rewritten in the form

$$
\int_{0}^{+\infty} R_{1}( \pm t \pm s) v(s) d s, \quad t \in \mathbb{R}_{+}
$$

with kernel

$$
R_{1}(t)=\int_{-\infty}^{+\infty} e^{i \mu t} Z_{1}(\mu) d \mu
$$

belonging to the space $L_{1}\left(\mathbb{R}, \mathbb{C}^{m} \times \mathbb{C}^{m}\right)$.

Proposition 7. Suppose that the operator function $S(i \mu)$ satisfies condition (A1). Then the operators

$$
D_{4}^{ \pm} v=Q_{ \pm}\left(\int_{-\infty}^{+\infty} e^{i \mu t}\left(S(i \mu) A^{n} P_{0}^{-1}(i \mu)\left(\int_{0}^{+\infty} e^{ \pm i \mu s} v(s) d s\right)\right) d \mu\right)
$$

are compact in $L_{2}\left(\mathbb{R}_{+}, \mathfrak{H}\right)$.

According to condition (A1), it suffices to establish that the operator

$$
D_{4, m}^{ \pm} v=Q_{ \pm}\left(\int_{-\infty}^{+\infty} e^{i \mu t}\left(Q_{m} S(i \mu) Q_{m} A^{n} P_{0}^{-1}(i \mu)\left(\int_{0}^{+\infty} e^{ \pm i \mu s} v(s) d s\right)\right) d \mu\right)
$$


is compact, because the sequence $D_{4, m}^{ \pm}$converges in the operator norm $\|\cdot \mid\| \|_{+}$to $D_{4}^{ \pm}$as $m \rightarrow \infty$. Indeed, since the Fourier transform is a unitary operator on $L_{2}(\mathbb{R}, \mathfrak{H})$, we have the inequality

$$
\left\|\left(D_{4}^{ \pm}-D_{4, m}^{ \pm}\right) v\right\|_{L_{2}} \leq \sup _{\mu \in \mathbb{R}}\left\|S(i \mu)-Q_{m} S(i \mu) Q_{m}\right\|\|v\|_{L_{2}} .
$$

In turn, the proof of the compactness of $D_{4, m}^{ \pm}$follows from Proposition 6. Indeed, by the well-known estimates (see [8]) of the operator function $P_{o}^{-1}(\lambda)$ and by condition (A1), we have

$$
\left\|Q_{m} S(i \mu) Q_{m} A^{n} P_{0}^{-1}(i \mu)\right\| \leq \frac{\text { const }}{|\mu|^{n}+1}, \quad|\mu| \rightarrow+\infty .
$$

The remaining part of the proof of Lemma 3 essentially reproduces the scheme of proof of the theorem on the $\Phi$-solvability of Wiener-Hopf integral equations on the half-line (see [10. Th. 2.1, pp. 13-16]). By analogy with [10, Th. 2.1], one can rewrite Eq. (12) in matrix form by identifying the space $L_{2}(\mathbb{R}, \mathfrak{H})$ with the orthogonal sum $L_{2}\left(\mathbb{R}_{+}, \mathfrak{H}\right) \oplus L_{2}\left(\mathbb{R}_{+}, \mathfrak{H}\right)$ and by assigning a vector function $\left(v_{1}, v_{2}\right)$ to each function $v \in L_{2}(\mathbb{R}, \mathfrak{H})$, where $v_{1}(t)=$ $v(t)(t \geq 0)$ and $v_{2}(t)=v(-t)(t>0)$. To prove that the operator $\mathfrak{N}$ (see Lemma 3) at the intersection of the first row and the first column in the resulting matrix is Fredholm, it suffices, by analogy with [10, Th. 2.1], to establish the unique solvability of Eq. (12) on the entire line in the space $L_{2}(\mathbb{R}, \mathfrak{H})$ and show that the off-diagonal entries of the matrix $D_{1}^{ \pm}+D_{2}^{ \pm}+D_{4}^{ \pm}$are compact operators on $L_{2}\left(\mathbb{R}_{+}, \mathfrak{H}\right)$.

The unique solvability of Eq. (12) in $L_{2}(\mathbb{R}, \mathfrak{H})$ is ensured by Lemma 2 , and the compactness of $D_{1}^{ \pm}, D_{2}^{ \pm}$, and $D_{4}^{ \pm}$follows from Propositions 3 and 7 and Lemma 4

Proposition 8. If the operator functions $K_{p}(t)$ range in the set of compact operators on $\mathfrak{H}$ and are Bochner integrable on $\mathbb{R}$, then the integral operator $D_{3}$ is compact in $L_{2}\left(\mathbb{R}_{+}, \mathfrak{H}\right)$.

Recall that the operator functions $K_{p}(t)$ for which the assumption of Proposition 5 holds satisfy relation (13).

Let $D_{3}=D_{3,1}+D_{3,2}+\cdots+D_{3, n}$, where

$$
D_{3, p} v=\int_{0}^{+\infty} K_{p}(t+s)\left(\int_{0}^{+\infty} A^{P} G_{0}^{(n-p)}(-s-\zeta) v(\zeta) d \zeta\right) d s .
$$

To prove Proposition 8 , note that, for each $m$, the operator

$$
D_{3, p, m} v=\int_{0}^{+\infty} Q_{m} K_{p}(t+s) Q_{m}\left(\int_{0}^{+\infty} A^{P} G_{0}^{(n-p)}(-s-\zeta) v(\zeta) d \zeta\right) d s
$$

is compact in $L_{2}\left(\mathbb{R}_{+}, \mathfrak{H}\right)$, because it is the composition of the compact operator with kernel $Q_{m} A^{p} G_{0}^{(n-p)}(-s-\zeta)$ (see Proposition 4) and the compact operator with kernel $Q_{m} K_{p}(t+s) Q_{m}, p=1,2, \ldots, n$ (see Proposition 4).

In turn, relation (13) implies that the sequence $D_{3, p, m}$ converges to $D_{3, p}$ in the operator norm $\|\cdot \cdot\|_{+}$. (See [3, Lemma 5] for details.) It follows that the operator $D_{3, p}$ is compact.

Proposition 9. Assume that the operator function $S(i \mu)$ satisfies condition (A1). Then the integral operator $D_{5}$ is compact in $L_{2}\left(\mathbb{R}_{+}, \mathfrak{H}\right)$.

The proof of Proposition 9 is similar to that of Proposition 8 and is based on the fact that the operators

$$
D_{5, m} v=-Q_{+}\left[F^{-1}\left[\left(Q_{m} S(i \mu) Q_{m}\right)\left[F\left[R Q_{+}\left[\int_{0}^{+\infty} Q_{m} A^{n} G_{0}(-s-\zeta) v(\zeta) d \zeta\right]\right]\right]\right]\right](t)
$$

are compact in $L_{2}\left(\mathbb{R}_{+}, \mathfrak{H}\right)$ for all $m$. This, in turn, follows from Propositions 4 and 6 and from the fact that the sequence $D_{5, m}$ converges in the operator norm $\|\cdot\| \|_{+}$to $D_{5}$. 
Lemma 5. Suppose that the operator function $S(i \mu)$ satisfies condition (A1) and the operator functions $K_{p}(t)$ satisfy the assumption of Proposition 5 . Then the integral operators $D_{6}$ and $D_{7}$ are compact in $L_{2}\left(\mathbb{R}_{+}, \mathfrak{H}\right)$.

The proof of the compactness of $D_{6}$ is completely similar to that of [3, Lemma 6] and uses the fact that the operator

$$
H_{m} v=Q_{m}\left(\sum_{m=1}^{k} \sum_{j=1}^{k} \alpha_{m j} A^{n-r_{j}} e^{\omega_{m} s A} \int_{0}^{+\infty} G_{0}^{\left(r_{j}\right)}(-\zeta) v(\zeta) b d \zeta\right), \quad s \in \mathbb{R}_{+},
$$

is compact in $L_{2}\left(\mathbb{R}_{+}, \mathfrak{H}\right)$.

The compactness of $D_{7}$ can readily be derived from the fact that the operators

$$
D_{7, m} v=Q_{+}\left(F^{-1}\left(\left(Q_{m} S(i \mu) Q_{m}\right) F\left(R H_{m} v\right)(\mu)\right)\right)
$$

are compact in $L_{2}\left(\mathbb{R}_{+}, \mathfrak{H}\right)$ and converge to $D_{7}$ in the operator norm $\||\cdot|\|_{+}$.

Let us complete the proof of Theorem 1. By Lemma 3, the operator $\mathfrak{N}$ is Fredholm. It is well known [11] that the sum of a Fredholm operator and a compact operator is a Fredholm operator. By Lemmas 3 and 5 and Propositions 8 and 9 , we obtain the desired assertion on the $\Phi$-solvability of the integral equation (11) in $L_{2}\left(\mathbb{R}_{+}, \mathfrak{H}\right)$.

The proof of Theorem 1 is complete.

We omit the proof of Theorem 2, because the papers [6] and [7] establish the wellposed solvability of equations containing variable coefficients and delay variables and are much more general than (5). The well-posed solvability is studied in the weighted (with exponential weight) Sobolev spaces $W_{2, \gamma}^{1}\left(\mathbb{R}_{+}, A\right)$ on the half-line. We also do not present the proof of Lemma 1, because a proof of close assertions can be found in [5] and [7.

Theorems 3 and 4 are easy corollaries of Theorems 1 . Since the operator $B$ is Fredholm, it suffices to establish that $\operatorname{Ker} B=\{0\}$.

In turn, the fact that, under the assumptions of Theorems 3 and 4 , the null space of $B$ is trivial can readily be established by integrating by parts in the corresponding quadratic form and by using the fact that the Fourier transform is a unitary operator on $L_{2}(\mathbb{R}, \mathfrak{H})$. Let us explain this in more detail.

Indeed, consider the following form on the solutions $v \in W_{2}^{1}\left(\mathbb{R}_{+}, A\right)$ of the homogeneous equation with $v(+0)=0$ :

$$
\begin{aligned}
\langle\mathfrak{U} v, A v\rangle_{L_{2}\left(\mathbb{R}_{+}, \mathfrak{H}\right)}= & \left\langle\frac{d v}{d t}, A v\right\rangle_{L_{2}\left(\mathbb{R}_{+}, \mathfrak{H}\right)}+\left\langle B_{1} A v, A v\right\rangle_{L_{2}\left(\mathbb{R}_{+}, \mathfrak{H}\right)} \\
& +\langle A v, A v\rangle_{L_{2}\left(\mathbb{R}_{+}, \mathfrak{H}\right)}+\left\langle\pi_{+}\left(F^{-1}(S(i \mu) F(R(A v)))\right), A v\right\rangle_{L_{2}\left(\mathbb{R}_{+}, \mathfrak{H}\right)} .
\end{aligned}
$$

Since

$$
\lim _{t \rightarrow+0}\left\|A^{1 / 2} v(t)\right\|=0
$$

for $v \in W_{2}^{1}\left(\mathbb{R}_{+}, A\right)$ and the Fourier transform is a unitary operator on $L_{2}\left(\mathbb{R}_{+}, \mathfrak{H}\right)$, we obtain, after integrating by parts in the first term on the right-hand side and then taking the real part in the last chain of equalities,

$$
\operatorname{Re}\left\langle B_{1} A v, A v\right\rangle_{L_{2}\left(\mathbb{R}_{+}, \mathfrak{H}\right)}+\langle A v, A v\rangle_{L_{2}\left(\mathbb{R}_{+}, \mathfrak{H}\right)}+\operatorname{Re}\langle S(i \mu) A \widehat{v}(i \mu), A \widehat{v}(i \mu)\rangle_{L_{2}\left(\mathbb{R}_{+}, \mathfrak{H}\right)} \geq 0 .
$$

It readily follows that $v \equiv 0$ and hence $\operatorname{Ker} B=0$.

To prove that $\operatorname{Ker} B=0$ in the case of Theorem 4, it suffices to consider the form $\left\langle\mathfrak{U} v, A^{2} v\right\rangle_{L_{2}\left(\mathbb{R}_{+}, \mathfrak{H}\right)}$ on the solutions $v(t)$ of the homogeneous equation with the condition $v(+0)=0$ or $v^{(1)}(+0)=0$. 
By integrating by parts in the expression $\left\langle\frac{d^{2} v}{d t^{2}}, A^{2} v\right\rangle_{L_{2}\left(\mathbb{R}_{+}, \mathfrak{H}\right)}$ and by taking into account the fact that the Fourier transform is unitary, we obtain

$$
\begin{aligned}
-\left\langle A v^{(1)}, A v^{(1)}\right\rangle_{L_{2}\left(\mathbb{R}_{+}, \mathfrak{H}\right)}+\operatorname{Re}\left\langle B_{2} A^{2} v, A^{2} v\right\rangle_{L_{2}\left(\mathbb{R}_{+}, \mathfrak{H}\right)}-\left\langle A^{2} v, A^{2} v\right\rangle_{L_{2}\left(\mathbb{R}_{+}, \mathfrak{H}\right)} & \\
& +\operatorname{Re}\left\langle S(i \mu) A^{2} \widehat{v}(i \mu), A^{2} \widehat{v}(i \mu)\right\rangle_{L_{2}\left(\mathbb{R}_{+}, \mathfrak{H}\right)} \leq 0 .
\end{aligned}
$$

We see that $v \equiv 0$ and hence $\operatorname{Ker} B=\{0\}$.

\section{Asymptotic Behavior of SOlutions and SOME SPECTRAL ISSUES}

In what follows, we study the asymptotic behavior of solutions of the equations considered in the present paper. We also give a number of results concerning the properties of the subsystem of elementary solutions corresponding to the characteristic numbers lying in the left half-plane and use them to establish results on the minimality of derived chains associated with these root vectors.

We use the definitions and notation given above.

We assume that the operator function $S(\lambda)$ satisfies the following condition:

(A3) There exists a $\delta>0$ such that the operator function $S(\lambda)$ is regular in the halfplane $\Pi_{\delta}^{-} \equiv\{\lambda: \operatorname{Re} \lambda<\delta\}$, ranges in the set of compact operators for $\lambda \in \Pi_{\delta}^{-}$, and satisfies the condition

$$
\|S(\lambda)\| \rightarrow 0 \quad \text { as }|\lambda| \rightarrow+\infty, \quad \lambda \in \Pi_{\delta}^{-} .
$$

Definition. An operator function $L(\lambda)$ belongs to the class $\mathfrak{M}_{\gamma}$ if the following conditions are satisfied:

1. There exists a $\gamma>0$ such that the spectrum of $L(\lambda)$ in the half-plane $\Pi_{\gamma}^{-} \equiv$ $\{\lambda: \operatorname{Re} \lambda<\gamma\}$ consists of isolated points that are poles of $L^{-1}(\lambda)$, the coefficients in the principal part of the Laurent expansions of $L^{-1}(\lambda)$ around these poles being finite rank operators.

2. For each $a>0$, there exists a $b>0$ such that

$$
\sup _{-a<\mu<0} \int_{b}^{+\infty}\left\|(\mu \pm i \nu)^{n-1} L^{-1}(\mu \pm i \nu)\right\|^{2} d \nu<+\infty .
$$

We denote Eq. (11) with $f(t) \equiv 0$ by (1) and the set of strong solutions $u(t)$ of Eq. (1P) such that $e^{\alpha t} u(t) \in L_{2}\left(\mathbb{R}_{+}, \mathfrak{H}\right)$ by $U_{\alpha}$.

Proposition 10. Suppose that the operator function $S(\lambda)$ satisfies condition (A3) and $K_{j}(t)=0, t>0, j=1,2, \ldots, n$.

The vector function

$$
y_{q, j, s}(t)=e^{\lambda_{q} t}\left(\frac{t^{s}}{s !} x_{q, j, 0}+\frac{t^{s-1}}{(s-1) !} x_{q, j, 1}+\cdots+x_{q, j, s}\right)
$$

is a solution of Eq. (1) if and only if the vectors $x_{q, j, 0}, x_{q, j, 1}, \ldots, x_{q, j, s}$ form a chain of root vectors corresponding to a characteristic number $\lambda_{q}$ of the pencil $L(\lambda)$ with $\operatorname{Re} \lambda_{q}<0$.

Theorem 5. Assume that the operator function $S(\lambda)$ satisfies condition (A3); the operator functions $K_{j}(t)$ additionally satisfy the conditions

$$
K_{j}(t)=0, \quad t>0, \quad e^{\beta t}\left\|K_{j}(-t)\right\| \in L_{2}\left(\mathbb{R}_{+}\right), \quad j=1,2, \ldots, n,
$$

for some $\beta \geq 0$, and $L(\lambda) \in \mathfrak{M}_{\varkappa}, 0<\varkappa<\min (\delta, \beta)$.

Then for each $\alpha \geq 0$ the set $U_{\alpha}$ is closed in the norm of $W_{2}^{n}\left(\mathbb{R}_{+}, A^{n}\right)$, and the subspace $U_{0}$ can be represented as the direct sum

$$
U_{0}=V_{\alpha}+U_{\alpha}
$$


where $V_{\alpha}$ is the finite-dimensional subspace spanned by the elementary solutions $y_{q, j, s}(t)$ corresponding to the characteristic numbers $\lambda_{q}$ of $L(\lambda)$ in the strip $\{\lambda:-\alpha \leq \operatorname{Re} \lambda<0\}$.

Corollary 1. Let the assumptions of Theorem [5 be satisfied. Then the elementary solutions $y_{q, j, s}(t)$ form a minimal system in $W_{2}^{n}\left(\mathbb{R}_{+}, A^{n}\right)$.

Let us present conditions sufficient for the pencil $L(\lambda)$ to belong to the class $\mathfrak{M}_{\varkappa}$.

Proposition 11. Suppose that the pencil $G(\lambda)$ can be represented in the form (44) and the operator functions $K_{j}(t), j=1,2, \ldots, n$, and $S(\lambda)$ satisfy the assumptions of Theorem 5. Then the operator function $L(\lambda)$ belongs to $\mathfrak{M}_{\varkappa}, 0<\varkappa<\min (\delta, \beta)$.

Theorem 5 and Proposition 11 readily imply the following assertion

Theorem 6. Suppose that the pencil $G(\lambda)$ can be represented in the form (4) and the operator functions $K_{j}(t), j=1,2, \ldots, n$, and $S(\lambda)$ satisfy the assumptions of Theorem 5. Then the assertion of Theorem 5 holds, and the elementary solutions $y_{q, j, s}(t) \in U_{0}$ form a minimal system in $W_{2}^{n}\left(\mathbb{R}_{+}, A^{n}\right)$.

Theorem 7 (The Phragmén-Lindelöf principle). Let the assumptions of Theorem 6 be satisfied, and let the operator $A^{-1}$ be of finite order (i.e., $\left.A^{-1} \in \sigma_{p}, 0<p<+\infty\right)$. Then the inclusion $u(t) \in \bigcap_{\alpha \geq 0} U_{\alpha}$ implies that $u(t) \equiv 0$.

Here we should note that assertions similar to Theorem $\square$ for solutions of functionaldifferential equations are often referred to as theorems on small solutions. Some comments and references to the literature where small solutions have been studied can be found in [31, 32, and [7, Ch. 2].

The following theorem states the well-posedness of the problem on a subspace. This theorem plays an important role when establishing minimality results for derived chains.

Theorem 8. Suppose that the assumptions of Theorem 7 are satisfied, the operator function $\left(L(\lambda) P_{0}^{-1}(\lambda)\right)^{-1}$ is bounded on the imaginary axis, and $\operatorname{det}\left\|\nu_{l, j}\right\|_{l, j=1}^{k} \neq 0$. Then there exists an $\eta \geq 0$ such that the inequality

$$
\|u\|_{W_{2}^{n}\left(\mathbb{R}_{+}, A^{n}\right)} \leq d\left(\sum_{j=1}^{k}\left\|u^{\left(r_{j}\right)}(+0)\right\|_{n-r_{j}-1 / 2}^{2}\right)^{1 / 2}
$$

with a constant $d$ independent of $u(t)$ holds for each solution $u(t) \in U_{\eta}$.

Theorem 9. Suppose that the assumptions of Theorem [6 are satisfied, $r_{1} \geq 1$, the operator function $\left(L(\lambda) P_{o}^{-1}(\lambda)\right)^{-1}$ is bounded on the imaginary axis, and $\operatorname{det}\left\|\nu_{l, j}\right\|_{l, j=1}^{k} \neq$ 0 . Then the vectors $z_{q, j, s}=\left(y_{q, j, s}^{\left(r_{1}-1\right)}(+0), \ldots, y_{q, j, s}^{\left(r_{k}-1\right)}(+0)\right)$, as well as the vectors $w_{q, j, s}=$ $\left(y_{q, j, s}^{\left(r_{1}\right)}(+0), \ldots, y_{q, j, s}^{\left(r_{k}\right)}(+0)\right)$ constructed according to (14) from the root vectors $x_{q, j, s}$ of $L(\lambda)$ corresponding to the characteristic numbers $\lambda_{q}$ with sufficiently large modulus in the left half-plane, form a minimal system in the space $\bigoplus_{m=1}^{k} \mathfrak{H}_{n-r_{m}-1 / 2}$.

Let us present a result refining Theorem 9 for the case in which $n=2$ and $k=1$.

Theorem 10. Suppose that the assumptions of Theorem 6 are satisfied with $n=2$, $\omega_{1}=-1, \omega_{2}=1, r_{1}=1, \operatorname{Re} B_{2} \leq 0, K_{j}(t) \equiv 0, j=1,2$, and the operator function $S(i \mu)$ additionally satisfies the condition

$$
\operatorname{Re}(S(i \mu) x, x) \leq 0
$$

for any $x \in \mathfrak{H}$ and $\mu \in \mathbb{R}$.

Then the vectors $z_{q, j, s}=y_{q, j, s}(+0)$ and $w_{q, j, s}=y_{q, j, s}^{(1)}(+0)$ constructed according to (14) from the root vectors $x_{q, j, s}$ of $L(\lambda)$ corresponding to the characteristic numbers in the left half-plane form a minimal system in $\mathfrak{H}_{1 / 2}$. 
Owing to the limited length of the paper, we only give sketches of the proofs and short explanations of the stated results.

The proofs of the assertions in this part of the paper are based on a representation of the Laplace transform of a strong solution of the homogeneous equation and on an estimate of the operator function $L^{-1}(\lambda)$. In our case, the Laplace transform has the form

$$
\hat{u}(\lambda)=L^{-1}(\lambda)(q(\lambda)+\eta(\lambda)+\mu(\lambda)+r(\lambda)), \quad \operatorname{Re} \lambda<\gamma_{1}<\varkappa,
$$

where

$$
\begin{aligned}
q(\lambda) & =\sum_{p=0}^{n-1} Q_{p}(\lambda) u^{(p)}(+0) \\
Q_{p}(\lambda) & =\lambda^{-(p+1)}\left(G(\lambda)-\sum_{s=0}^{p} \lambda^{s} G_{n-s} A^{n-s}\right) \\
\eta(\lambda) & =-\frac{1}{2 \pi i} \int_{-\infty}^{+\infty} \frac{S\left(\gamma_{1}+i \nu\right) A^{n} \hat{u}\left(\gamma_{1}+i \nu\right)}{\left(\gamma_{1}+i \nu\right)-\lambda}, \\
\mu(\lambda) & =\sum_{p=1}^{n} \int_{0}^{+\infty} e^{-\lambda s}\left(\int_{-\infty}^{+\infty} K_{p}(\xi) e^{-\lambda \xi} d \xi\right) A^{p} u^{(n-p)}(s) d s, \\
r(\lambda) & =\sum_{p=1}^{n} T_{p}(\lambda)\left(\sum_{j=0}^{n-p-1} \lambda^{j} u^{(n-p-j-1)}(+0)\right) \\
T_{p}(\lambda) & =\int_{-\infty}^{0} e^{-\lambda t} K_{p}(t) d t .
\end{aligned}
$$

By using the assumptions made about the operator functions $K_{p}(t)$ and $S(\lambda)$, one can prove by a straightforward verification that the vector function

$$
\xi(\lambda)=q(\lambda)+\eta(\lambda)+r(\lambda)+\mu(\lambda)
$$

is holomorphic in the half-plane $\left\{\lambda: \operatorname{Re} \lambda<\gamma_{1}\right\}$ and admits the estimates

$$
\left\|\frac{d^{k}}{d \lambda^{k}} \xi(\lambda)\right\| \leq(1+|\lambda|)^{n-k-1}\left(1+\frac{1}{\sqrt{2\left(\gamma_{1}-\operatorname{Re} \lambda\right)}}\right)\|u\|_{W_{2}^{n}}, \quad k=0,1, \ldots .
$$

By Keldysh's well-known result [12], the principal part of the vector function $\hat{u}(\lambda)$ in a neighborhood of a pole $\lambda=\lambda_{q}$ has the form

(17) Principal part of $(\hat{u}(\lambda))$

$$
=\sum_{(j)}\left\{c_{q, j, r_{j}} \frac{x_{q, j, 0}}{\lambda-\lambda_{q}}+\cdots+c_{q, j, 0}\left(\frac{x_{q, j, 0}}{\left(\lambda-\lambda_{q}\right)^{r_{j}+1}}+\frac{x_{q, j, 1}}{\left(\lambda-\lambda_{q}\right)^{r_{j}}}+\cdots+\frac{x_{q, j, r_{j}}}{\left(\lambda-\lambda_{q}\right)}\right)\right\},
$$

where the coefficients $c_{q, j, r}\left(r=0,1, \ldots, r_{j}\right)$ are determined as follows:

$$
\begin{aligned}
c_{q, j, 0} & =\left(\xi\left(\lambda_{q}\right), z_{q, j, 0}\right), \quad \ldots, \\
c_{q, j, r_{j}} & =\left(\xi\left(\lambda_{q}\right), z_{q, j, r_{j}}\right)+\frac{1}{1 !}\left(\frac{d \xi}{d \lambda}\left(\lambda_{q}\right), z_{q, j, r_{j-1}}\right)+\cdots+\frac{1}{r_{j} !}\left(\frac{d^{r_{j}}}{d \lambda^{r_{j}}} \xi\left(\lambda_{q}\right), z_{q, j, 0}\right) .
\end{aligned}
$$

Here $x_{q, j, 0}, \ldots, x_{q, j, r_{j}}$ is the canonical system of root vectors of $L(\lambda)$ corresponding to the characteristic number $\lambda=\lambda_{q}$, and $z_{q, j, 0}, \ldots, z_{q, j, r_{j}}$ is the system of root vectors of $\left(L(\lambda) A^{-n}\right)^{*}$ corresponding to the characteristic number $\lambda=\bar{\lambda}_{q}$.

Proposition 10 can be proved by a straightforward verification. 
We should point out that Theorem 5 can be obtained from the representation (15) of the Laplace transform $\hat{u}(\lambda)$ for $\operatorname{Re} \lambda<\gamma_{1}<\varkappa$, the estimates (16), and also the representation (17). The main assertion of Theorem 5 on the closedness of the subspace $U_{\alpha}$ is a consequence of the above-mentioned results, because if the principal part of the Laplace transforms $\hat{u}_{m}(\lambda)$ of functions $u_{m}(t) \in U_{\alpha}$ in a neighborhood of a pole $\lambda_{q}$ is zero, then so is the principal part of the limit function $\hat{u}(\lambda)=\lim _{m \rightarrow \infty} \hat{u}_{m}(\lambda)$.

Theorem [6 readily follows from Theorem 5 and the well-known estimates (see [12]) of the pencil $G^{-1}(\lambda)$ for the case of formulas (4).

The proof of Theorem 7 is fairly traditional. Under the assumption that $A^{-1} \in \sigma_{p}$ $(0<p<+\infty)$ and $u(t) \in \bigcap_{\alpha \geq 0} U_{\alpha}$, one can prove on the basis of Radzievskii's results in [24] that the transform $\hat{u}(\lambda)$ extends to be an entire function of order $\leq p$. Indeed, the function $\hat{u}(\lambda)$ belongs to the Hardy space in the right half-plane as the Laplace transform of a function in $L_{2}\left(\mathbb{R}_{+}, \mathfrak{H}\right)$, while in the left half-plane one has the representation (15) for $\hat{u}(\lambda)$, which, in view of Radzievskii's above-mentioned results in 24, implies that the order of $\hat{u}(\lambda)$ does not exceed $p$. An application of the Phragmén-Lindelöf theorem to the function $\hat{u}(\lambda)$ in the left half-plane completes the proof of Theorem 7 .

Let us discuss this in more detail.

Consider the domain

$$
\Psi_{\delta, \rho} \equiv\{\lambda: \operatorname{Re} \lambda<0\} \backslash\left(\bigcup_{m=1}^{k}\left\{\lambda:\left|\arg \lambda-\arg \omega_{m}\right| \leq \delta\right\} \cup\{\lambda:|\lambda| \geq \rho\}\right) .
$$

It follows from the well-known estimates

$$
\left\|\lambda^{p} A^{n-p} P_{0}^{-1}(\lambda)\right\| \leq \mathrm{const}, \quad\left\|P_{0}^{-1}(\lambda)\right\| \leq \frac{\text { const }}{|\lambda|^{n}+1}, \quad \lambda \in \Psi_{\delta, \rho},
$$

the relations (see [9] and [24]) that

$$
\lim _{\substack{|\lambda| \rightarrow+\infty \\ \lambda \in \Psi_{\delta, \rho}}}\left\|\lambda^{p} B_{n-p} A^{n-p} P_{0}^{-1}(\lambda)\right\|=0, \quad p=1,2, \ldots, n,
$$

and the conditions imposed on the operator functions $K_{p}(t)$ and $S(\lambda)$ that

$$
\lim _{\substack{|\lambda| \rightarrow+\infty \\ \lambda \in \Psi_{\delta, \rho}}}\left\|\lambda^{p} T_{n-p}(\lambda) A^{n-p} P_{0}^{-1}(\lambda)\right\|=0, \quad p=1,2, \ldots, n, \lim _{\substack{|\lambda| \rightarrow+\infty \\ \lambda \in \Psi_{\delta, \rho}}}\left\|S(\lambda) A^{n} P_{0}^{-1}(\lambda)\right\|=0 .
$$

These formulas imply that, for each sufficiently small $\delta$, there exists a $\rho>0$ such that the operator function $L^{-1}(\lambda)$ is holomorphic in the domain $\Psi_{\delta, \rho}$ and admits the estimate

$$
\left\|L^{-1}(\lambda)\right\| \leq \frac{\text { const }}{|\lambda|^{n}+1} .
$$

Then the representation (15) and the estimate (16) for $k=0$ imply the inequality

$$
\|\hat{u}(\lambda)\| \leq \frac{\text { const }}{|\lambda|+1}, \quad \lambda \in \Psi_{\delta, \rho} .
$$

From the last inequality, in view of the fact that the vector function $\hat{u}(\lambda)$ is an entire function of order $\leq p$, we find that the Phragmén-Lindelöf theorem applies for $0<\delta<$ $\pi / \rho$ in each of the sectors $\left\{\lambda:\left|\arg \lambda-\arg \omega_{j}\right|<\delta\right\}$. By this theorem, $\hat{u}(\lambda)$ is bounded in each of these sectors. In turn, since $\hat{u}(\lambda)$ is an entire vector function, it follows from the estimate (18) that $\hat{u}(\lambda) \equiv 0$ and hence $u(t) \equiv 0$.

The proofs of Theorems 8,10 are completely similar to that in the polynomial case (see [2]-[3]) and are based on Theorems [5] and 7

Indeed, an analysis of the proofs of assertions in [2] and [3] similar to Theorems 8 and 9 shows that, for them to be true, it suffices to require that the original problem be Fredholm and use the Phragmén-Lindelöf principle. 
Let us discuss this in more detail. The sets $\left\{U_{\alpha}\right\}_{\alpha \geq 0}$ form a chain of nested subspaces, $U_{\alpha_{2}} \subseteq U_{\alpha_{1}}$ for $\alpha_{1}>\alpha_{2}$; these subspaces are closed by Theorem 6, and their intersection is empty by Theorem 7 (the Phragmén-Lindelöf principle), $\bigcap_{\alpha \geq 0} U_{\alpha}=\{0\}$. Since the operator $B$ is Fredholm (Theorem 1), it follows that so is the operator $T$ defined by

$$
T u=\left(u^{\left(r_{1}\right)}(+0), u^{\left(r_{2}\right)}(+0), \ldots, u^{\left(r_{k}\right)}(+0)\right)
$$

and viewed as an operator acting from the solution subspace $U_{0} \subset W_{2}^{n}\left(\mathbb{R}_{+}, A^{n}\right)$ into the space $\bigoplus_{m=1}^{k} \mathfrak{H}_{n-r_{m}-1 / 2}$. Since $\operatorname{Ker} T$ is finite-dimensional, we see that there exists an $\eta \geq 0$ such that $U_{\eta} \cap \operatorname{Ker} T=\{0\}$. By Theorem [6] $U_{\eta}$ is a (closed) subspace of $W_{2}^{n}\left(\mathbb{R}_{+}, A^{n}\right)$, and hence $T U_{\eta}$ is a (closed) subspace of $\bigoplus_{m=1}^{k} \mathfrak{H}_{n-r_{m}-1 / 2}$. In fact, this is a consequence of the following simple proposition.

Proposition 12. Let $T$ be a Fredholm operator from a Hilbert space $X$ to a Hilbert space $Y$, and let $\mathfrak{L} \subset X$ be a (closed) subspace such that $\operatorname{Ker} T \cap \mathfrak{L}=\{0\}$.

Then $T \mathfrak{L}$ is a subspace of $Y$ as well.

Consider the restriction $T_{\eta}$ of $T$ to the subspace $U_{\eta} \subseteq U_{0}$. Then $T_{\eta}$ is a one-to-one mapping of $U_{\eta}$ onto the subspace $T U_{\eta}$ (by the choice of $\eta$ ). By the Banach inverse operator theorem, $T_{\eta}$ has a bounded inverse $T_{\eta}^{-1}$, which just means that Theorem 8 holds.

\section{SOME REMARKS AND COMMENTS}

Theorem 1 generalizes the corresponding assertions in 3 and 4 . The papers [3] and [4] consider Eq. (11) with $S(i \mu) \equiv 0$ and $K_{j}(t) \equiv 0, j=1,2, \ldots, n-1$. The proof of Theorem 1 is largely similar to that of [3, Th. 1].

The polynomial case was considered in numerous papers. Of the ones closest to the present paper, we mention [1], 2], 8], 9], [15, [25], and [26].

Let us now clarify how the results of the present paper are related to those in [5]-[7]. Consider an operator function $S(i \mu)$ of the form

$$
S(i \mu)=\sum_{j=1}^{n} e^{-\mu h_{j}} B_{j}, \quad \mu \in \mathbb{R} .
$$

If the operators $B_{j}, j=1,2, \ldots, n$, are compact, then $S(i \mu)$ ranges in the algebra of compact operators on $\mathfrak{H}$ satisfying condition (A1).

The operator function $S(i \mu)$ corresponds to the expression

$$
\sum_{j=1}^{n} B_{j}\left(S_{h_{j}} u\right)(t)
$$

considered in [5]- 7].

Note, however, that we a priori assume in the present paper that the solutions are bounded as $t \rightarrow+\infty$ (more precisely, belong to the space $W_{2}^{n}\left(\mathbb{R}_{+}, A^{n}\right)$ ).

It is of interest to note that $S(i \mu)$ also satisfies condition (A1) for negative $h_{j}, j=$ $1,2, \ldots, n$, which corresponds to the case of an advance equation. However, we should point out that the problem posed for the advance equation is not the initial value problem (infamous for its peculiarities, in particular, ill-posedness; see [17]) but rather a problem with a priori restrictions on the growth of the solution at infinity.

Let us briefly discuss other results given in the paper.

Theorem [5 on the dichotomy of solutions is an analog of assertions earlier known for ordinary differential equations with operator coefficients (see [1, [2], 26], and [30]). In turn, assertions about the existence or nonexistence of nontrivial solutions decaying 
more rapidly than any exponential (the Phragmén-Lindelöf principle) were established for ordinary differential equations with operator coefficients in [1, 2], 26, and [30].

Informally speaking, Theorem 5 implies that problem (11), (6) is well-posed on the subspace $U_{\eta}(\eta \geq 0)$, whereas it may remain ill-posed on $U_{0}$.

Let us briefly discuss spectral issues. The completeness and minimality of full and partial systems of root vectors of polynomial operator pencils was studied by numerous authors. We only mention the papers [1], 2], 8], [9], [1]-[13], [15], and [25]-28].

There are quite a few papers studying the completeness of part of the system of root vectors for operator functions with more complicated (nonpolynomial) dependence on the spectral parameter. Of the closest results, we note those due to Radzievskii [19, [22], 24] (see also the bibliographies therein).

We point out, however, that our method for studying minimality issues is substantially different from Radzievskii's approach. The most important thing in our approach is the study of geometric properties of exponential (elementary) solutions, while the results concerning the properties of derived chains are obtained as corollaries by applying the trace operator.

For polynomial operator pencils and the corresponding equations, this approach was developed and used several times by Shkalikov [26].

The present paper is a natural development of the author's earlier results [1, 22 for polynomial operator pencils of Keldysh type.

\section{REFERENCES}

[1] V. V. Vlasov, Multiple minimality of a part of a system of root vectors of $M$. V. Keldysh's pencils, Dokl. Akad. Nauk SSSR 263 (1982), no. 6, 1289-1293; English transl., Sov. Math. Dokl. 25 (1982), 511-514. MR653219 (83h:47016)

[2] V. V. Vlasov, The k-fold minimality of root vectors of Keldysh-type pencils, Mat. Fiz. (1982), no. 32, 80-89. (Russian) MR711715 (85j:47014)

[3] V. V. Vlasov, Solvability of boundary value problems for a class of integro-differential equations on the half-axis, Differentsial'nye Uravneniya 25 (1989), no. 9, 1589-1599; English transl., Differential Equations 25 (1989), no. 9, 1124-1132 (1990). MR1024676 (90m:45020)

[4] V. V. Vlasov, On the solvability of a class of functional-differential equations on the semi-axis and some spectral problems, Dokl. Akad. Nauk SSSR 319 (1991), no. 1, 22-26; English transl., Sov. Math. Dokl. 44 (1992), no. 1, 14-19. MR.1140826 (92m:47156)

[5] V. V. Vlasov, On the solvability and properties of solutions of functional-differential equations in a Hilbert space, Mat. Sb. 186 (1995), no. 8, 67-92; English transl., Sb. Math. 186 (1995), no. 8, 1147-1172. MR1357357 (96g:34121)

[6] V. V. Vlasov, On the solvability and estimates for the solutions of functional-differential equations in Sobolev spaces, Tr. Mat. Inst. Steklova 227 (1999), 109-121; English transl., Proc. Steklov Inst. Math. (1999), no. 4 (227), 104-115. MR1784309 (2001j:35265)

[7] V. V. Vlasov and D. A. Medvedev, Functional-differential equations in Sobolev spaces and related problems in spectral theory, Sovrem. Mat. Fundam. Napravl. 30 (2008), 3-173; English transl., J. Math. Sci. (N. Y.) 164 (2010), no. 5, 659-841. MR2472270 (2009m:35528)

[8] M. G. Gasymov, The multiple completeness of part of the eigen- and associated vectors of polynomial operator bundles, Izv. Akad. Nauk Armjan. SSR Ser. Mat. 6 (1971), no. 2-3, 131-147. (Russian) MR0298446 (45:7498)

[9] M. G. Gasymov, The solvability of boundary value problems for a class of operator-differential equations, Dokl. Akad. Nauk SSSR 235 (1977), no. 3, 505-508; English transl., Sov. Math. Dokl. 18 (1977), 943-947 (1978). MR0461184 (57:1169)

[10] I. Ts. Gokhberg and M. G. Krein, Systems of integral equations on the half-line with kernels depending on the difference of the arguments, Uspekhi Mat. Nauk (N.S.) 13 (1958), no. 2 (80), 3-72. (Russian) MR0102720(21:1506)

[11] I. C. Gohberg [I. Ts. Gokhberg] and M. G. Krein, Introduction to the theory of linear nonselfadjoint operators in Hilbert space, Nauka, Moscow, 1965; English transl., Transl. of Math. Monographs, Amer. Math. Soc., Providence, RI, 1969. MR0246142 (39:7447) 
[12] M. V. Keldysh, The completeness of eigenfunctions of certain classes of nonselfadjoint linear operators, Uspekhi Mat. Nauk 26 (1971), no. 4 (160), 15-41; English transl., Russian Math. Surveys 26 (1971), no. 4, 15-44. MR0300125(45:9173)

[13] V. B. Lidskii, Summability of series in terms of the principal vectors of nonselfadjoint operators, Tr. Mosk. Mat. Obs. 11 (1962), 3-35. (Russian) MR0144213 (26:1760)

[14] J.-L. Lions and E. Magenes, Problèmes aux limites non homogenes et applications. Vol. 1, Dunod, Paris, 1968; English transl., Springer, New York, 1972. MR0247243 (40:512)

[15] S. S. Mirzoev, The double completeness of a part of the eigen- and associated vectors of polynomial operator pencils of fourth order, Izv. Akad. Nauk Azerbaijan. SSR Ser. Fiz.-Tekhn. Mat. (1974), no. 6, 37-42. (Russian) MR0374964 (51:11160)

[16] S. G. Mikhlin, Linear partial differential equations, Vyssh. Shkola, Moscow, 1977. (Russian) MR510535 (80a:35001)

[17] A. D. Myshkis, Linear differential equations with retarded argument, Moscow, Nauka, 1972. (Russian) MR0352648(50:5135)

[18] G. V. Radzievskii, Multiple completeness of the root vectors of an $M$. V. Keldysh pencil perturbed by an operator-valued function analytic in the disc, Mat. Sb. (N.S.) 91 (133) (1973), no. 3 (7), 310-335; English transl., Math. USSR-Sb. 20 (1973), no. 3, 323-347. MR0341148 (49:5898)

[19] G. V. Radzievskii, The basis property of derived chains, Izv. Akad. Nauk SSSR Ser. Mat. 39 (1975), no. 5, 1182-1218; English transl., Math. USSR-Izv. 9 (1975), no. 5 1119-1154. MR0407634 $(53: 11406)$

[20] G. V. Radzievskii, The completeness of derived chains, Mat. Sb. (N.S.) 100(142) (1976), no. 1, 37-58; English transl., Math. USSR-Sb. 29 (1976), no. 1, 35-54. MR0415377 (54:3465)

[21] G. V. Radzievskii, Completeness of derived chains corresponding to boundary value problems on a semiaxis, Ukrain. Mat. Zh. 31 (1979), no. 4, 407-416; English transl., Ukrainian Math. J. 31 (1979), no. 4, 319-327. MR543435 (81c:34015b)

[22] G. V. Radzievskii, Bases consisting of derivatives of chains corresponding to boundary value problems, Dokl. Akad. Nauk SSSR 251 (1980), no. 2, 283-287. (Russian) MR.565495 (83c:47021)

[23] G. V. Radzievskii, Asymptotic behavior of distributions of characteristic numbers of operator functions analytic in a sector, Mat. Sb. (N.S.) 112 (154) (1980), no. 3 (7), 396-420; English transl., Math. USSR-Sb. 40 (1981), no. 3, 363-385. MR582191 (81i:47007)

[24] G. V. Radzievskii, The problem of completeness of root vectors in the spectral theory of operatorvalued functions, Uspekhi Mat. Nauk 37 (1982), no. 2 (224), 81-145; English transl., Russ. Math. Surveys 37 1982, no. 2, 91-164. MR650759 (84b:47023)

[25] A. A. Shkalikov, Some questions of the theory of polynomial operator pencils, Uspekhi Mat. Nauk 38 (1983), no. 3 (231), 189-190; English transl., Russ. Math. Surveys 38 (1983), no. 3, $151-152$. MR:704652 (85c:47016)

[26] A. A. Shkalikov, Elliptic equations in Hilbert space and associated spectral problems, Tr. sem. im. I. G. Petrovskogo (1989), no. 14, 140-224; English transl., J. Math. Sci. 51 (1990), no. 4, 2399-2467. MR $1001359(90 \mathrm{~g}: 47090)$

[27] A. A. Shkalikov, Operator-differential equations on the half-axis and related spectral problems for selfadjoint operator pencils, Dokl. Akad. Nauk SSSR 276 (1984), no. 2, 309-314; English transl., Sov. Math. Dokl. 29 (1984), 511-516. MR.745036(86c:34122)

[28] A. A. Shkalikov, Operator-differential equations on the half-axis and related spectral problems for polynomial operator pencils, Uspekhi Mat. Nauk 39 (1984), no 4, 106. (Russian)

[29] E. M. Stein, Singular integrals and differentiability properties of functions, Princeton University Press, Princeton, NJ, 1970. MR0290095 (44:7280)

[30] S. Agmon and L. Nirenberg. Properties of solutions of ordinary differential equations in Banach Space, Comm. Pure Appl. Math. 16 (1963), 121-239. MR0155203 (27:5142)

[31] P. D. Lax, Phragmén-Lindelöf theorem in harmonic analysis and its application to some questions in elliptic equations, Comm. Pure Appl. Math. 10 (1957), 361-389. MR0093706 (20:229)

[32] J. Hale and S. M. Verduyn Lunel, Introduction to functional-differential equations, Appl. Math. Studies, vol. 99, Springer-Verlag, New York, 1993. MR1243878 (94m:34169)

Faculty of Mechanics and Mathematics, Moscow State University, 1 Leninskie Gory, 119991

Moscow, Russian Federation

E-mail address: vikvvlasov@rambler.ru 